\title{
Sun Tracker Robotic Arm Optical Distance Measurement Evaluation at Different Positions Using Six Sigma Tools
}

\author{
Roland Szabo and Aurel Gontean, Member, IEEE
}

\begin{abstract}
This paper presents an optical distance evaluation solution, for a sun tracker robotic arm, with the help of Six Sigma. With the help of statistical tools any measurement system can be evaluated and a measurement system analysis (MSA) can be easily made. Like every measurement system, optical distance measurement can have errors. Six Sigma tools can evaluate the measurement system and can give useful data about its accuracy. Sometimes measurement system evaluation is done twice. First, initial tests are done, after some fine tuning and error correction is performed, and finally a repeated test is done, to show that the measurement errors were corrected.
\end{abstract}

Index Terms-cameras, databases, distance measurement, data processing, stereo vision.

\section{INTRODUCTION}

T HISpaper presents an evaluation tool of an optical distance measurement system. Optical distance measurement can have errors, apparently larger than traditional systems $[1,2]$. Using the Six Sigma $(6 \sigma)$ tools, we can ensure that optical distance measurement is accurate, having high precision at smaller and bigger distances too [18].

In today's industrialized world, robots are the key elements which makes possible to build products in big volume and with a high-quality standard to cover the needs of today's consumer world [3, 4, 14, 15]. Robots need to measure distances to know their position in space and the distance to the manipulated object [5]. They must use optical distance measurement methods to ensure flexibility [6]. Optical distance measurement with the usage of stereo cameras [16] is one of the most common ways to achieve high precision distance measurement for industrial robots [7].

Measurement system analysis (MSA) is often performed to ensure that the system behaves as desired [10]. First, a pre-test is done, where the measurement system errors are measured [11]. After, some corrections are made, and is checked if the changes really reduced the measurement errors [12].

This paper was submitted for review on February 2, 2019

This work was supported by a grant of the Romanian National Authority for Scientific Research and Innovation, CNCS/CCCDI - UEFISCDI, project number PN-III-P2-2.1-PED-2016-0074, within PNCDI III.

R. Szabo and A. Gontean are with Politehnica University Timisoara, Faculty of Electronics, Telecommunications and Information Technologies, Applied Electronics Department, V. Parvan Av., No. 2, 300223, Timisoara, Romania (e-mail: roland.szabo@upt.ro, aurel.gontean@upt.ro).

\section{PROBlem Formulation}

In the laboratory we had access to an educational/industrial robotic arm (Fig. 1), which we needed to program to track the sun [18]. The demand was to know its position in space and the distance to the manipulated object to implement automated software which can control it to move autonomously [18]. The optical distance measurement was the best solution to obtain the position of the key parts of the robotic arm in space [8]. We evaluated the optical distance measurement against the distances measured with laser. The optical distance measurement had some errors. We had to measure somehow if these errors are acceptable, if some corrections are needed, or if we need to use another distance measurement method [9].

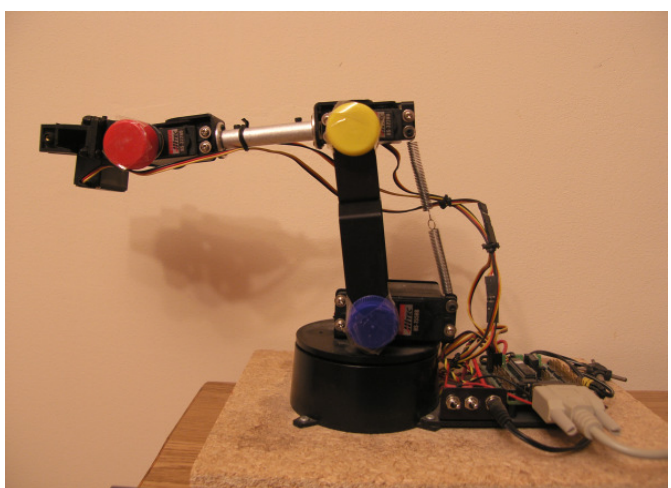

Fig. 1. The normal probability plot, for the actual real distance and the distance computed, by the system using the cameras.

On Fig. 2 there can be seen the optical distance measurement method with two cameras (stereo cameras).

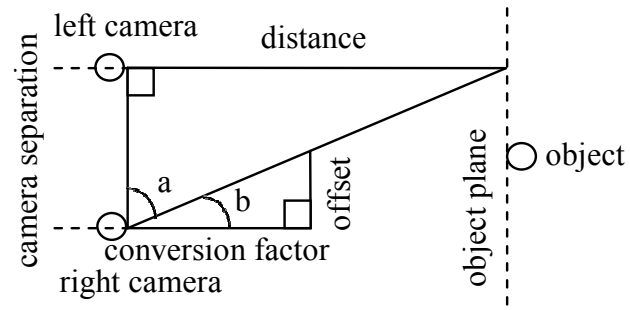

Fig. 2. Distance computation using two video cameras (stereo cameras). 
The camera separation and initial distance from the base of the robotic arm is measured previously with laser. These values are hardcoded in the automated distance measurement software, made by the authors, in $\mathrm{C}++$ programming language, for the robotic arm. The automated distance measurement system can compute the distances to each colored bottle cap (blue, yellow and red) at the robotic arm's joints. The colored bottle caps are recognized using color filtering.

On the $0 x$ and $0 y$ axes the distance can be computed easily based on a $2 \mathrm{D}$ coordinate system based on the pixels from one camera. To compute the distance on the $0 z$ axis (in $3 \mathrm{D}$ ) the following formulas were used.

On equation (1) can be seen the tangent of the $a$ and $b$ angles.

$$
\left\{\begin{array}{l}
\operatorname{tg}(a)=\frac{\text { distance }}{\text { camera separation }} \\
\operatorname{tg}(b)=\frac{\text { offset }}{\text { conversion factor }}
\end{array}\right.
$$

The initial offset can be computed from the $x$ coordinates of the left and right camera, as shown on equation (2).

$$
\text { initial off set }=\left|x_{i_{R}}-x_{i_{L}}\right|
$$

The conversion factor can be computed as shown on equation (3).

$$
\text { conversion factor }=\frac{\text { initial of } f \text { set } \times \text { initial distance }}{\text { camera } \text { separation }}
$$

The final offset can be computed from the $x$ coordinates of the left and right camera, as shown on equation (4).

$$
\text { final offset }=\left|x_{f_{R}}-x_{f_{L}}\right|
$$

The final distance can be computed as shown on equation (5).

$$
\text { final distance }=\frac{\text { converison } \text { factor } \times \text { camera separation }}{\text { final offset }}
$$

The novelty of the paper is measuring movement distances for a robotic arm's gripper, during movement (dynamically), with the usage of two cameras (stereo cameras) in real-time.

To have a robotic arm which can move freely, to execute any given task, as it was programmed previously (statically), the optical distance measurement must be very precise. To test the robustness of the optical distance measurement, the system must be tested as any industrial process. The best way to test this is to create a measurement system analysis (MSA) using Six Sigma tools.

\section{PROBLEM SOLUTION}

The optical distance measurement method was tested using Six Sigma tools [17]. Six Sigma represents a set of methods and tools which are used to improve the process. Six Sigma methods try to improve the quality of a process by finding and eliminating the root causes of defects. It uses a set of quality management tools, mainly statistical tools. The Six Sigma tools were implemented using graphs in Minitab statistical software [18].

These tools can show if the measurements are accurate enough and if some fine tuning is needed [13].

As shown on Table I., the system was tested with measurements at different distances. The range of values used for the measurements is between $100 \mathrm{~mm}$ and $3000 \mathrm{~mm}$, with $100 \mathrm{~mm}$ step. The real values, measured by laser, and the distance measured and computed by the system with the cameras are very close. There are two cameras, they are placed near each other, and the distance is computed with stereo triangulation. We computed also an error delta, the difference between the actual distance value and the value measured by the camera system. The low sample size is not a problem for the Six Sigma tools, because it can be used always normal probability plot instead of histograms.

TABLE I

Distance Measurement Using Cameras at DifFerent Distances [Mm] AND THE MEASUREMENT ERROR DELTA [MM]

\begin{tabular}{rrr}
\hline \hline Real Distance $[\mathrm{mm}]$ & $\begin{array}{c}\text { Computed Distance } \\
{[\mathrm{mm}]}\end{array}$ & Error Delta $[\mathrm{mm}]$ \\
\hline 100 & 99 & 1 \\
200 & 202 & -2 \\
300 & 303 & -3 \\
400 & 395 & 5 \\
500 & 502 & -2 \\
600 & 598 & 2 \\
700 & 699 & 1 \\
800 & 797 & 3 \\
900 & 904 & -4 \\
1000 & 1005 & -5 \\
1100 & 1101 & -1 \\
1200 & 1204 & -4 \\
1300 & 1298 & 2 \\
1400 & 1402 & -2 \\
1500 & 1494 & 6 \\
1600 & 1599 & 1 \\
1700 & 1701 & -1 \\
1800 & 1802 & -2 \\
1900 & 1905 & -5 \\
2000 & 2005 & -5 \\
2100 & 2105 & -5 \\
2200 & 2202 & -2 \\
2300 & 2302 & -2 \\
2400 & 2404 & -4 \\
2500 & 2496 & 4 \\
2600 & 2598 & -6 \\
2700 & 2697 & 4 \\
2800 & 2796 & 302 \\
2900 & 3006 & \\
3000 & & -6 \\
& & \\
\hline \hline
\end{tabular}

\section{EVALUATION}

In Fig. 3 we present a normal probability plot with a $95 \%$ confidence interval of the two measurements, the real distance and the measured distance with the camera system. Please note that these graphs overlap almost perfectly, so the measured distance, does not differ very much from the actual distance. The Anderson-Darling (AD)value is 0.321 , and the $\mathrm{p}$-value (the $\mathrm{p}$-value or probability value is the probability for a specific statistical model, when the null hypothesis is true) is 
Sun Tracker Robotic Arm Optical Distance Measurement Evaluation at Different Positions Using Six Sigma Tools
0.514 , so it is higher than $\alpha=0.05$ (the significance level $\alpha$ is the probability of making the wrong decision, when the null hypothesis is true), so the null hypothesis is not rejected; thus, we can say that the measured distance values do not differ too much from each other.

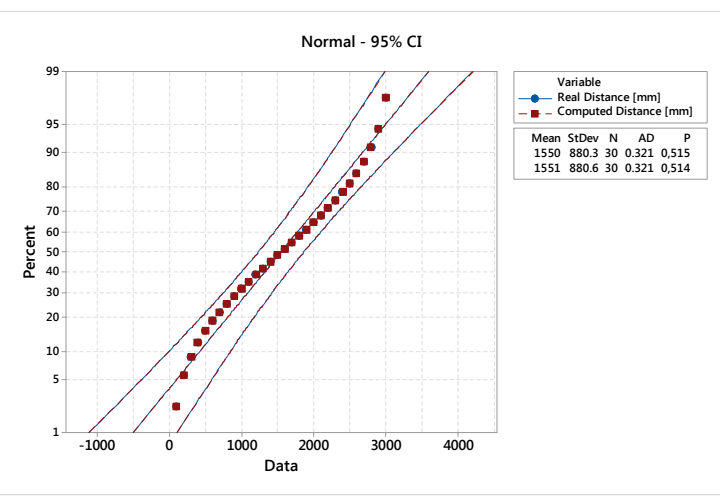

Fig. 3. The normal probability plot, for the actual real distance and the distance computed, by the system using the cameras.

In Fig. 4 we see the normal probability plot for absolute error delta with a confidence interval of $95 \%$. The mean for the absolute error delta is $3.033 \mathrm{~mm}$. The standard deviation is $1.586 \mathrm{~mm}$. The $\mathrm{AD}$ value is 1.261 , and the value is $\mathrm{p}<0.005$, which is lower than 0.05 , so the null hypothesis is rejected. This means that the absolute error delta values differ from each other, which could be expected, because for absolute error delta is a computed value, not a measured one. Each value is transformed to a positive error, all errors are added and stacked, and all errors are over 0 , even if the computed distance is higher or lower than the real distance. The mean of the absolute error has the value just over $3 \mathrm{~mm}$.

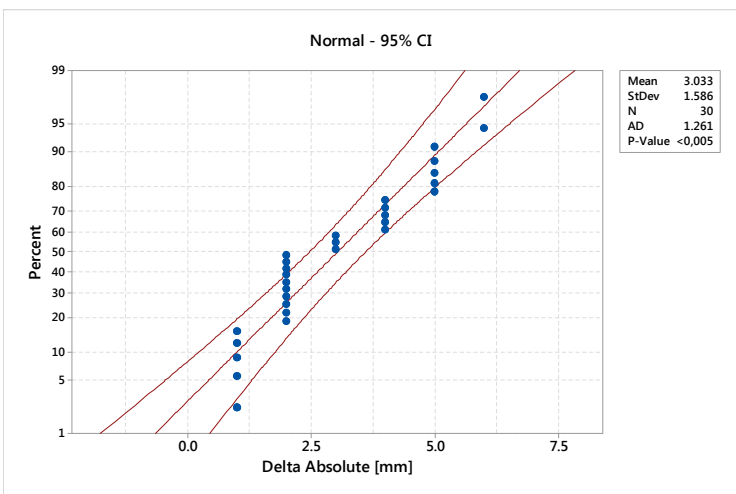

Fig. 4. The normal probability plot created for the absolute error delta.

In Fig. 5 we can see the fitted line plot, which is a method of the regression analysis. The computed distance is presented as a function of the actual real distance. The values are obtained first from the actual real distance values and then from the computed distance values. The standard deviation of these values is $3.42693 \mathrm{~mm}$. The R-Square (R-Sq) and RSquare adjusted (R-Sq (adj)) values are $100 \%$, which shows that the responses can be perfectly predicted by knowing the input values. This means that if we know the actual real distance, we can obtain the distance computed by the algorithm using video cameras. For regression analysis we obtained an empirical formula presented in the graph and shown in equation (6).

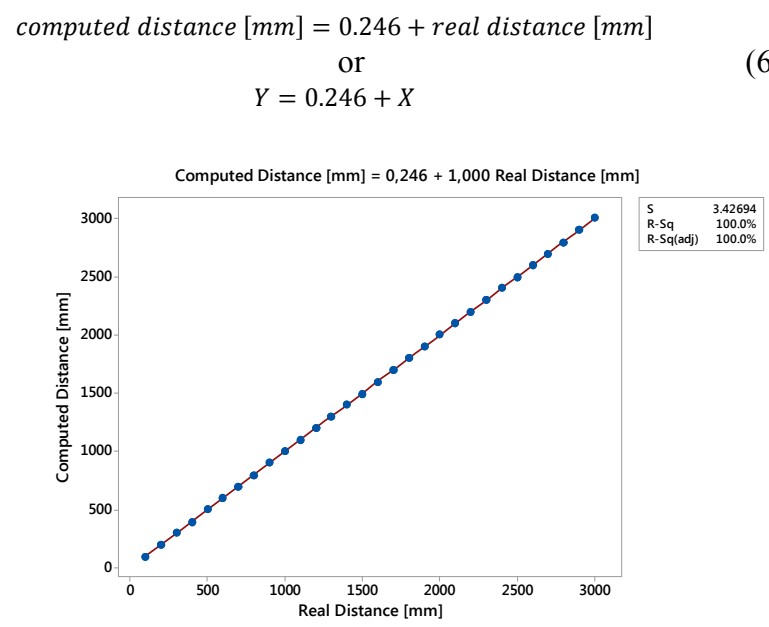

Fig. 5. Regression analysis, for actual real distance and the distance computed, by the system using cameras.

In Fig. 6 we show a more detailed analysis of regression, where, versus graphs are observed, indicating that there are no problems with the regression model, because the values are spread randomly above and below 0 . Also, on the graph we can see a red dot, representing a high residual value, at the (-6, 1500) coordinate. This means that for the measurement of $1500 \mathrm{~mm}$, the largest error delta is $6 \mathrm{~mm}$.

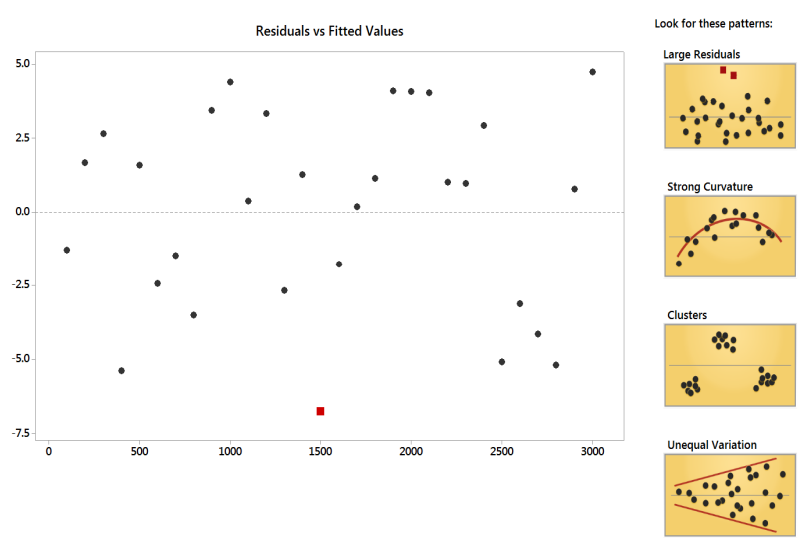

Fig. 6. The graph of residuals versus fitted values, for the actual real distance and the distance computed, by the system using cameras.

In Fig. 7 we show the residual values obtained after the fitted line plot. We can see that the $\mathrm{AD}$ value is 0.549 , and the p-value is 0.145 , this is higher than $\alpha=0.05$, the null hypothesis is not rejected. Thus, we can say that the residual values do not differ too much from each other. The histogram does not look like a Gaussian distribution, but for such a small number of values it cannot be expected a better result. The versus graphs (versus fits and versus order) show a large distribution of the values, which is normal and expected. 

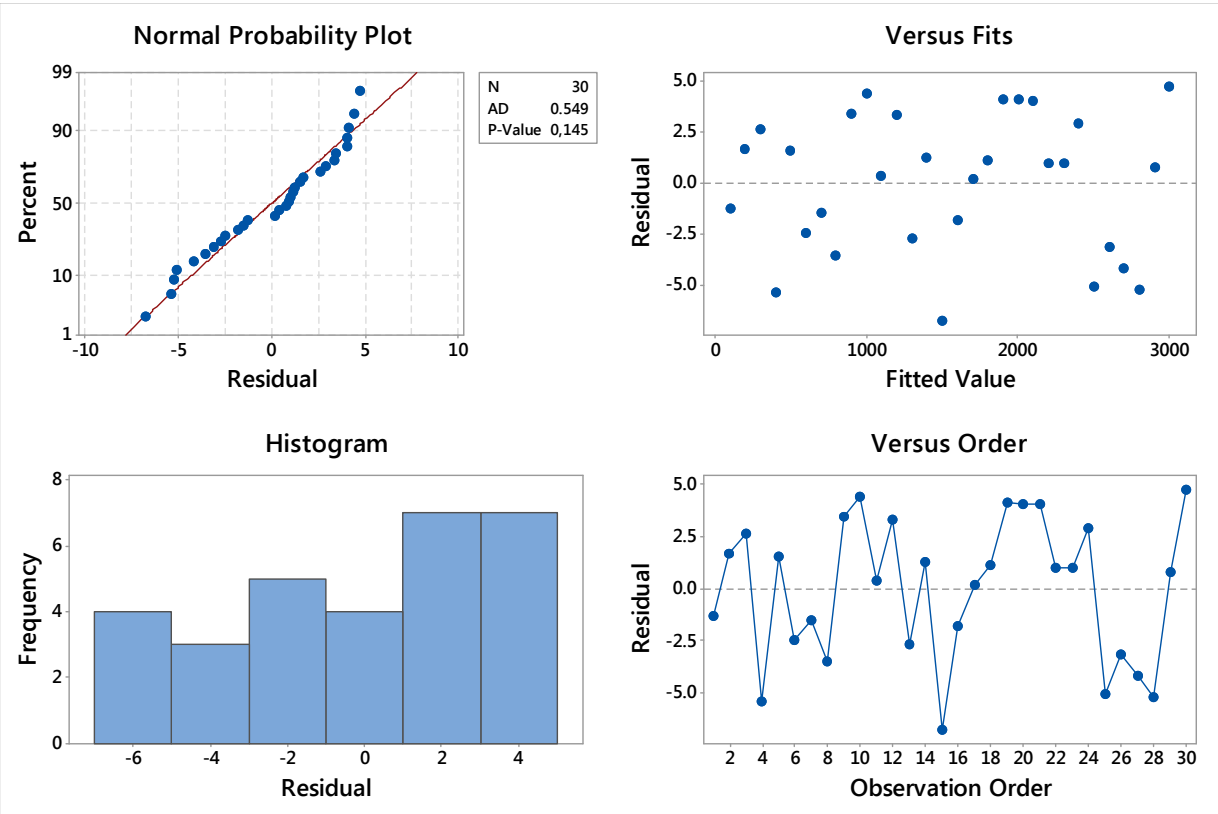

Fig. 7. Analysis of the residual values, for the distance computed, by the system using cameras.

In Fig. 8 we continue the regression analysis between the actual real distance and the distance computed by the algorithm for measuring distances using cameras. For each input value $\mathrm{x}$ (actual real distance) there is a prediction of $\mathrm{y}$ (the computed distance) using a prediction interval of $95 \%$ for $\alpha=0.05$.

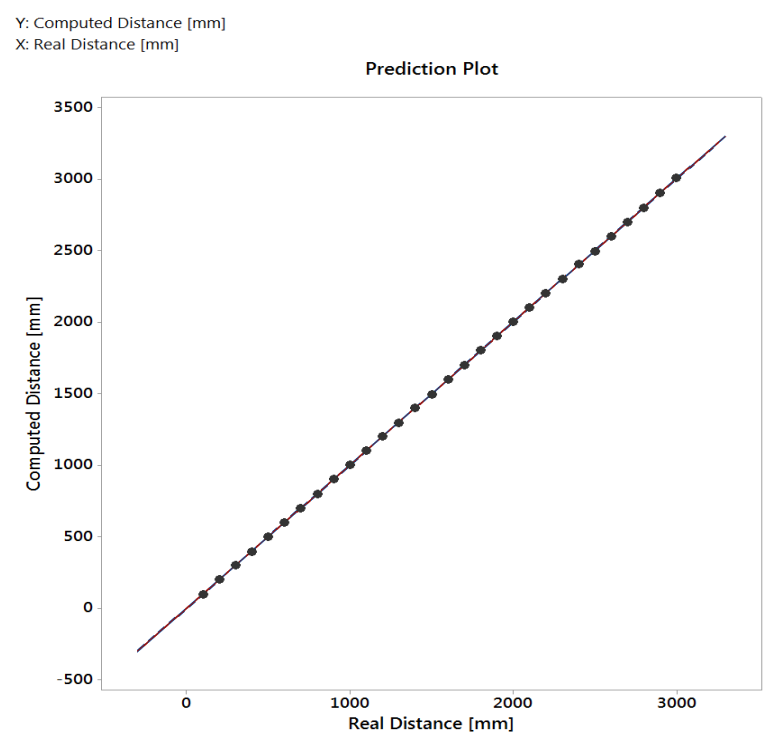

Fig. 8. The prediction plot, for the actual real distance and the distance computed, by the system using cameras.

In Fig. 9 we continue again the regression analysis, highlighting certain important values. We can see the RSquare (R-Sq) value adjusted to $100 \%$, which shows that the output values (computed distance) can be predicted 100\% from the input values (actual real distance). The residual standard deviation is $3.427 \mathrm{~mm}$ in the linear model and in the alternative quadratic model is $3.468 \mathrm{~mm}$; these values are very good and there were expected. On the graph we can observe the empirical formula obtained by the regression analysis and the highest residual value of $6 \mathrm{~mm}$ when measuring the distance of $1500 \mathrm{~mm}$.

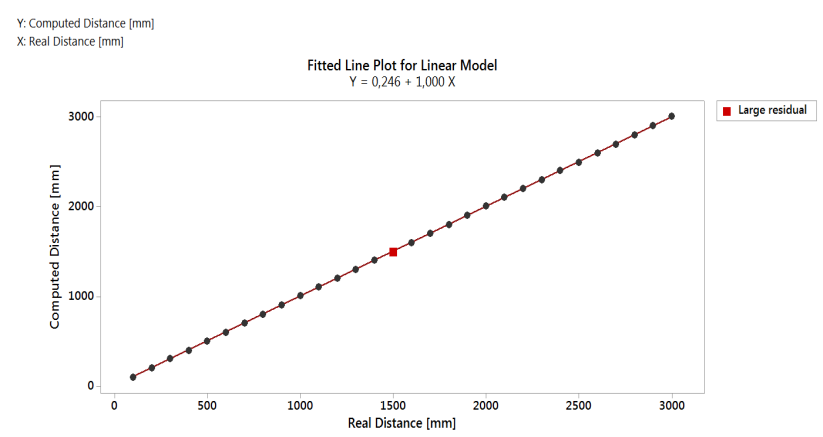

Fig. 9. The fitted line plot for linear model, for the actual real distance and the distance computed, by the system using cameras.

In Fig. 10 we present the summary report of the regression analysis. We can see that $\mathrm{p}<0.001$, which is lower than $\alpha=$ 0.05 , so the null hypothesis is rejected: This means that the measured values differ enough from each other, to form a mathematical relationship between the computed distance (Y) and the actual real distance $(\mathrm{X})$. The model variation, the RSquare (R-Sq) is $100 \%$, therefore the output values (computed distance) can be predicted 100\% from the input values (actual real distance), in other words the variation of the model is very small or non-existent, or the computed values with the camera system are very close to the actual real values measured with laser. There is a perfect correlation between the computed distance (Y) and the actual real distance (X) using the empirical equation obtained by the regression analysis. 


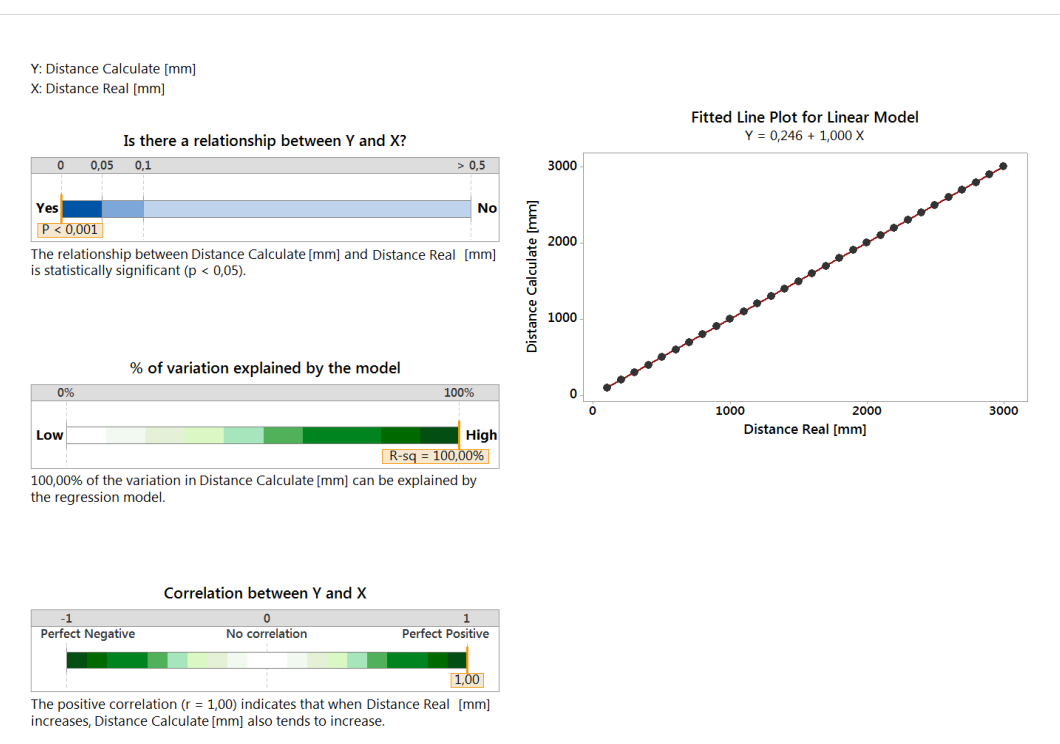

Fig. 10. Summary report of the regression analysis, for the actual real distance and the distance computed, by the system using cameras.

In Fig. 11 we show the capability analysis for 30 error deltas. The individual value and moving range (I-MR) charts are within limits. The histogram has somehow a Gaussian tendency but for a histogram, 30 values are not enough (better use normal probability plot). On the histogram we observe that there are no values where the error delta is 0 , which means there is no measurement without error, but the errors are not too high. On the normal probability plot, the $\mathrm{AD}$ value is 0.643 and the $p$-value is 0.084 , so this is higher than $\alpha=0.05$, so the null hypothesis is not rejected. This means that the error deltas do not differ too much between each other. The most important values are at the conclusions: the standard deviation is $2.965 \mathrm{~mm}$ for the subgroup and $3.38 \mathrm{~mm}$ for all the measurements. The process capability index for subgroup: $\mathrm{C}_{\mathrm{pk}}$ $=1.38$, over $4 \sigma\left(\right.$ where $\left.C_{p k}=1.33\right)$, which is a very good result in a system of this kind. The performance for subgroup: $C_{p}=$ 1.46 , the performance overall the data: $P_{p}=1.28$ and the process capability index overall the data: $\mathrm{P}_{\mathrm{pk}}=1.21$, all values are very close $4 \sigma$, which is a very good result for a system running under normal conditions. For parts per million (PPM), or the reported error to one million measurements is 20.21 for the subgroup and 171.11 for all the measurements, these values are also very good.
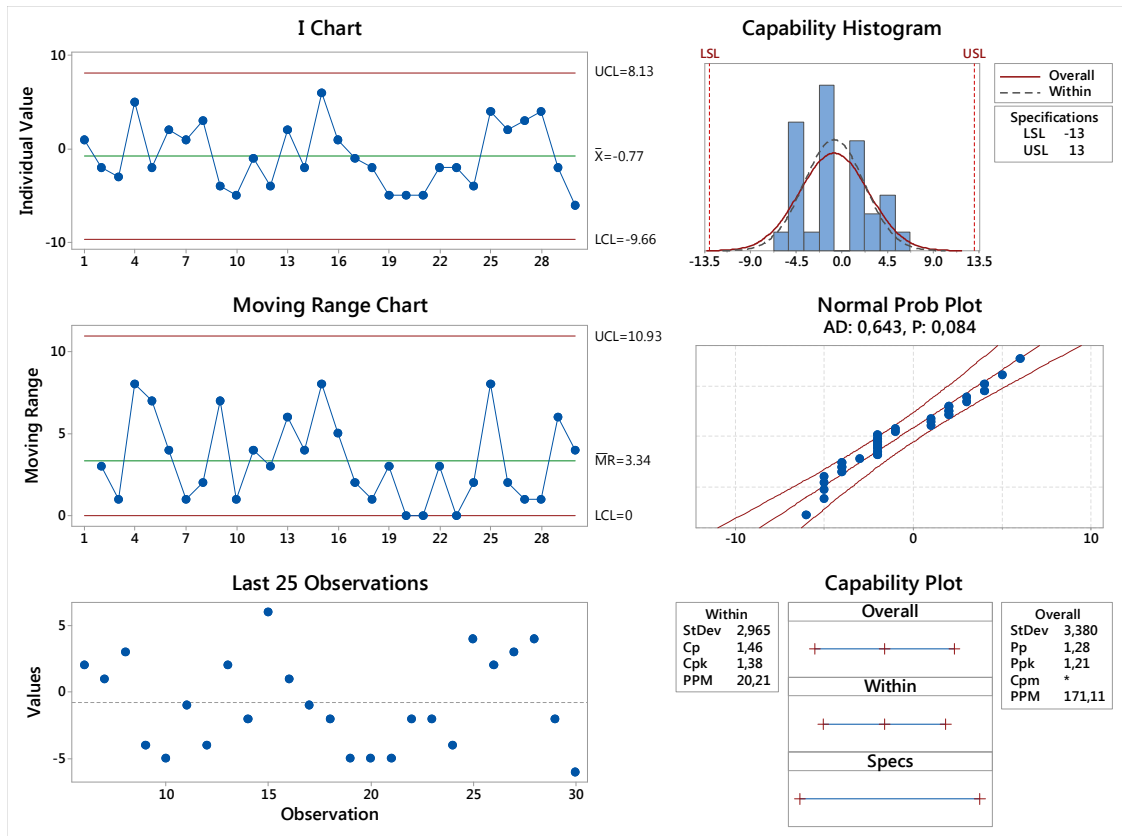

Fig. 11. The process capability analysis made for the error delta 
In Fig. 12 we continue the capability analysis for the error delta. We can see the individual value and moving range (IMR) charts which are in limits. The normality (AndersonDarling) test is passed with the p-value of 0.084 , which is greater than $\alpha=0.05$, so the null hypothesis is not rejected. Thus, we can say that the error delta values do not differ too much between each other.
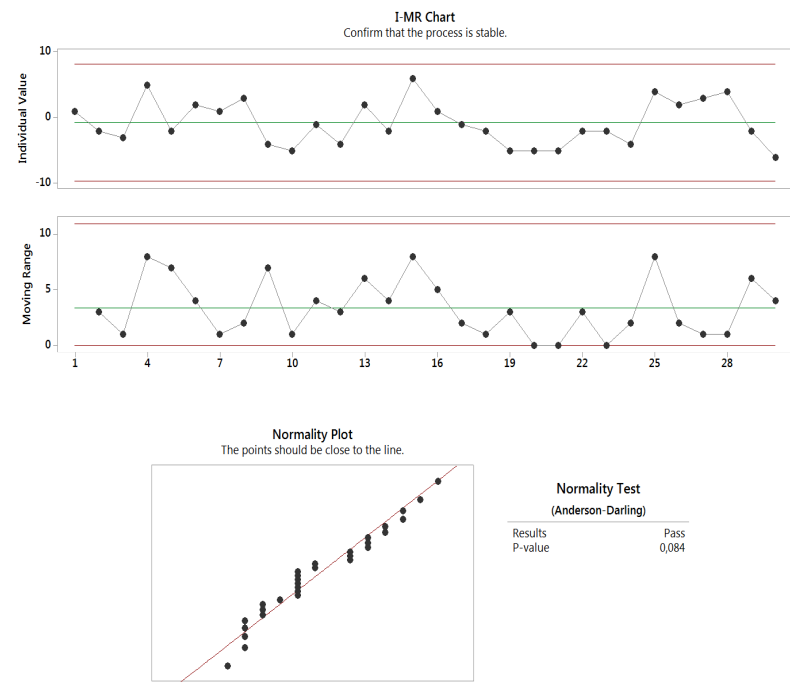

Fig. 12. The I-MR (individual value - moving range) analysis and the normality test made for the error delta.

In Fig. 13 we observe the fluctuation of the error delta, measured in $\mathrm{mm}$, as a function of the actual real distance, measured with laser in $\mathrm{m}$. We can see a tendency of fluctuation of higher values, for the small distances, under 1 $\mathrm{m}$, a tendency of fluctuation of proportional values, for the average distances, between $1 \mathrm{~m}$ and $2 \mathrm{~m}$, and a tendency of fluctuation of lower values, for the higher distances, between 2 $\mathrm{m}$ and $3 \mathrm{~m}$. This was expected, as this chart represents proportionality, so after this graph it can be said that the error deltas are mostly constant throughout all the distance ranges. This graph is a qualitative rather than a quantitative one; it shows the errors as the measurement distance is increased.

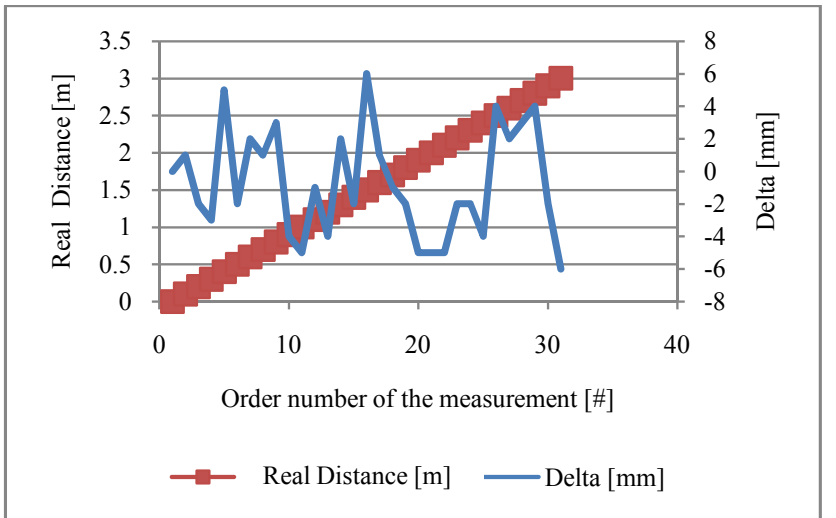

Fig. 13. Graphic representation of the actual real distance $[\mathrm{m}]$ and the error of measurement (error delta) [mm] on the same graph.

\section{CONCLUSION}

As it was seen an optical distance measurement system, with stereo cameras, used for a sun tracker robotic arm, was evaluated using Six Sigma tools.

The Six Sigma tools materialized by the graphs in Minitab showed that the optical distance measurement with video cameras is accurate enough and there is no need for fine tuning or replacing with other distance measurement method. This means that the measurement system analysis (MSA) has good results, $4 \sigma$ accuracy which for real processes is very good $(6 \sigma$ is the theoretically ideal process, not existent in real life). Knowing that the MSA had good results we can say that the industrial robots can precisely detect their position in space and the distance to the manipulated object just by using optical distance measurement using video cameras.

\section{ACKNOWLEDGMENT}

This work was supported by a grant of the Romanian National Authority for Scientific Research and Innovation, CNCS/CCCDI - UEFISCDI, project number PN-III-P2-2.1PED-2016-0074, within PNCDI III.

\section{REFERENCES}

[1] Wong Guan Hao, Yap Yee Leck, Lim Chot Hun, "6-DOF PC-Based Robotic Arm (PC-ROBOARM) with efficient trajectory planning and speed control," in Proc. 4th International Conference On Mechatronics, Kuala Lumpur, Malaysia, May 17-19, 2011, pp. 1-7.

[2] Woosung Yang, Ji-Hun Bae, Yonghwan Oh, Nak Young Chong, BumJae You, Sang-Rok Oh, "CPG based self-adapting multi-DOF robotic arm control," in Proc. International Conference on International Conference on Intelligent Robots and Systems, Taipei, Taiwan, October 18-22, 2010, pp. 4236-4243.

[3] E. Oyama, T. Maeda, J. Q. Gan, E. M. Rosales, K. F. MacDorman, S. Tachi, A. Agah, "Inverse kinematics learning for robotic arms with fewer degrees of freedom by modular neural network systems," in Proc. International Conference on International Conference on Intelligent Robots and Systems, August 2-6, 2005, pp. 1791-1798.

[4] N. Ahuja, U. S. Banerjee, V. A. Darbhe, T. N. Mapara, A. D. Matkar, R.K. Nirmal, S. Balagopalan, "Computer controlled robotic arm," in Proc. 16th IEEE Symposium on Computer-Based Medical Systems, New York, NY, USA, June 26-27, 2003, pp. 361-366.

[5] M. H. Liyanage, N. Krouglicof, R. Gosine, "Design and control of a high performance SCARA type robotic arm with rotary hydraulic actuators," in Proc. Canadian Conference on Electrical and Computer Engineering, St. John's, NL, USA, May 3-6, 2009, pp. 827-832.

[6] M. Mariappan, T. Ganesan, M. Iftikhar, V. Ramu, B. Khoo, "A design methodology of a flexible robotic arm vision system for OTOROB," in Proc. 2nd International Conference on Mechanical and Electrical Technology, Singapore, September 10-12, 2010, pp. 161-164.

[7] Guo-Shing Huang, Xi-Sheng Chen, Chung-Liang Chang, "Development of dual robotic arm system based on binocular vision," in Proc. International Automatic Control Conference, Nantou, Taiwan, December 2-4, 2013, pp. 97-102.

[8] N. C. Orger, T. B. Karyot, "A symmetrical robotic arm design approach with stereo-vision ability for CubeSats," in Proc. 6th International Conference on Recent Advances in Space Technologies, Istanbul, Turkey, June 12-14, 2013, pp. 961-965.

[9] F. Medina, B. Nono, H. Banda, A. Rosales, "Classification of Solid Objects with Defined Shapes Using Stereoscopic Vision and a Robotic Arm," in Proc. Andean Region International Conference, Cuenca, Spain, 2012, pp. 226.

[10] M. Puheim, M. Bundzel, L. Madarasz, "Forward control of robotic arm using the information from stereo-vision tracking system," in Proc. 14th International Symposium on Computational Intelligence and Informatics, Budapest, Hungary, November 19-21, 2013, pp. 57-62. 
[11] T. P. Cabre, M. T. Cairol, D. F. Calafell, M. T. Ribes, J. P. Roca, "Project-Based Learning Example: Controlling an Educational Robotic Arm with Computer Vision," IEEE Revista Iberoamericana de Tecnologias del Aprendizaje, vol. 8, issue 3, 2013, pp. 135-142.

[12] G. S. Gupta, S. C. Mukhopadhyay, M. Finnie, "WiFi-based control of a robotic arm with remote vision," in Proc. IEEE Instrumentation and Measurement Technology Conference, Singapore, May 5-7, 2009, pp. $557-562$.

[13] Haoting Liu, Wei Wang, FengGao, Zhaoyang Liu, Yuan Sun, Zhenlin Liu, "Development of Space Photographic Robotic Arm based on binocular vision servo," in Proc. Sixth International Conference on Advanced Computational Intelligence, Hangzhou, China, October 1921, 2013, pp. 345-349.

[14] Wen-Chung Chang, Chih-Wei Cho, "Automatic Mobile Robotic Manipulation with Active Eye-to-Hand Binocular Vision," in Proc. 33rd Annual Conference of the IEEE Industrial Electronics Society, Taipei, Taiwan, November 5-8 2007, 2007, pp. 2944-2949.

[15] P. C. Nunnally, J. M. Weiss, "An inexpensive robot arm for computer vision applications," in. Proc. IEEE Energy and Information Technologies in the Southeast, Columbia, SC, USA, vol. 1, April 9-12, 1989, pp. 1-6.

[16] T. Kizaki, A. Namiki, "Two ball juggling with high-speed hand-arm and high-speed vision system," in Proc. IEEE International Conference on Robotics and Automation, Saint Paul, MN, USA, May 14-18, 2012, pp. 1372-1377.

[17] Atarod Goudarzlou, Tan Kay Chuan "Using six sigma in new service development," in Proc. International Conference on Service Systems and Service Management, 2008, pp. 1-6.

[18] R. Szabo, A. Gontean, "Sun tracker robotic arm optical distance measuring algorithm evaluation using Six Sigma methods," in Proc. International Conference on Environment and Electrical Engineering, Milan, Italy, June 6-9, 2017, pp. 1439-1444.

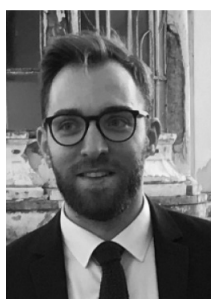

Roland Szabo was born in Timisoara, Romania on April 14, 1986. He received the B.Sc., M.Sc. and Ph.D. degrees from the Politehnica University Timisoara in 2009, 2011 and 2015, respectively. In 2009 he joined the Applied Electronics Department of the Politehnica University Timisoara where he is now a Lecturer. $\mathrm{He}$ is a hardware engineer, mainly with software tasks, developing test system software, at Continental Automotive Romania, located in Timisoara, since 2009. He is a LabWindows/CVI (ANSI C programming language) and NI TestStand (test sequencer language) industrial test software design trainer at Honeywell Life Safety, located in Lugoj, Romania since 2012.He got a Design for Six Sigma Green Belt (DfSS) certificate from Innovensys Germany in 2015, a LabVIEW Basics I \& II certificate from National Instruments Hungary in 2009. He is the author of 4 books, has 67 papers, 3 patent proposals and is a team member in 5 research grants.

His research interests are: robotics, computer communication interfaces, measuring equipment, software drivers, servers, mobile programming, web programming, programming languages, operating systems, communication systems, and embedded systems.

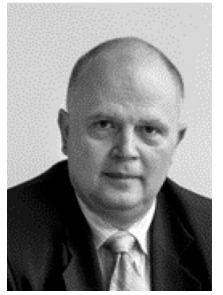

Aurel Gontean (M'05) was born in Petrosani, Romania on June 27, 1961. He received the Eng. and Ph.D. degrees from the Politehnica University Timisoara in 1986 and 1998, respectively.

$\mathrm{He}$ is a professor since 2005 in the Applied Electronics Department of the Politehnica University Timisoara. He is a $\mathrm{PhD}$ Advisor since 2008 (field: Electronics and Telecommunications). From 2004 till 2012 he was vice dean of the Electronics and Telecommunications Faculty, Timisoara, Romania. He is the author of 8 books, has over 150 papers, 3 patent proposals and has over 30 research grants and contracts. His research interests are in renewable energies, smart cities and memristors. He is also a visiting professor at the Loerrach University, Germany since 2009. He has been appointed as EU expert for evaluating project proposals for the Scientific Research Fund, Sofia, Bulgaria, from 2008 to 2009 . He was recipient of the IEEE International Conference on Intelligent Data Acquisition and Advanced Computing Systems Best Paper Award in 2011. 